\title{
A Simple Method for Enhancing the SNR in Direct Detection $\varphi$-OTDR based Distributed Acoustic Sensors
}

\author{
Faruk Uyar ${ }^{1}$, Tolga Kartaloglu ${ }^{1}$, Canberk Unal ${ }^{1}$, Ekmel Özbay $^{1,2,3}$ and Ibrahim Ozdur ${ }^{1,4}$ \\ ${ }^{1}$ Nanotechnology Research Center, Bilkent University, Ankara, Turkey \\ ${ }^{2}$ Department of Electrical and Electronics Engineering, Bilkent University, Ankara, Turkey \\ ${ }^{3}$ Department of Physics, Bilkent University, Ankara, Turkey \\ ${ }^{4}$ Department of Electrical and Electronics Engineering, Abdullah Gül University, Kayseri, Turkey \\ *Corresponding author: faruk.uyar@bilkent.edu.tr
}

\begin{abstract}
A simple and effective method for increasing the SNR and range in $\varphi$-OTDR based direct-detection distributed acoustic sensors is proposed and experimentally verified. The proposed method results in $\sim 11 \mathrm{~dB}$ SNR improvement at $66 \mathrm{~km}$. () 2020 The Author(s)
\end{abstract}

\section{Introduction}

Distributed acoustic sensor (DAS) systems have attracted considerable attention in the last few decades due to their applications in the security and monitoring of large-distance perimeters such as pipelines, borderlines, railways and so on [1]. These systems are based on optical time domain reflectometry (OTDR) technique that enables the characterization and fault location in optical fiber transmission cables. Unlike conventional OTDR systems, which can only provide the loss and reflection profile of the optical link, $\varphi$-OTDR (phase - OTDR) based DAS systems are capable of measuring the phase changes along the fiber by using a highly coherent (narrow linewidth) light source.

High signal-to-noise ratio (SNR) and large dynamic range is needed for long operating distances and reliable vibration measurements. Various studies have been undertaken to increase SNR and range of the DAS systems such as coherent detection [2], Brillouin amplification [3], Raman assistance [4], hybrid mechanisms [5] and so on. These approaches, however, significantly raise the system cost and complexity comparing to the direct detection approach. In this study, we propose a practical and effective method to improve the dynamic range and SNR performance of the $\varphi$-OTDR based DAS systems, which can be adopted by the direct detection architecture with a simple modification.

\section{Details of the method}

The proposed method suggests that the backscattered signal from the close-in distances and far away distances of the sensing fiber is handled separately for obtaining the spatial profile of the entire fiber. The power of the signal which is close to the launching-end is attenuated whereas that of the signal towards the far-end of the fiber is kept at maximum level. With this method, the signal saturation at close-in channels as well as the weakening of the signal at far away channels are eliminated. The proposed scheme utilizes an electronic variable optical attenuator (EVOA) for this purpose which is controlled by a control signal synchronized to the acousto-optic modulator (AOM). The EVOA attenuates the signals coming from the close-in channels only; and allows other signals to pass through without attenuation. The Rayleigh backscattering trace is observed by interrogating a $\sim 41-\mathrm{km}$ test fiber with pulses whose width is $100 \mathrm{~ns}$ and period is $500 \mu \mathrm{s}$. The EVOA is driven by a square control signal with a period of $500 \mu \mathrm{s}$ (same as the period of interrogation pulses) and a duty cycle of $30 \%$ (inset of Fig. 1b).

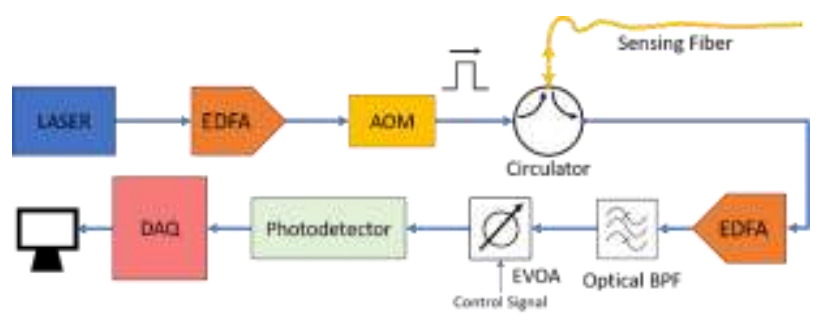

(a)

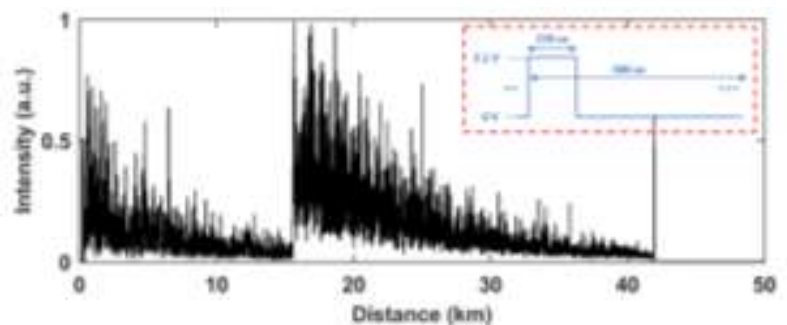

(b)

Fig. 1. a) Experimental set-up, b) Rayleigh backscattered trace in the proposed EVOA scheme

\section{Experimental results}

To test the SNR improvement of the proposed method, a test set-up with different lengths of the fibers and six piezoelectric transducers (PZT) is used with a total fiber length of $66 \mathrm{~km}$. Test set-up, shown in Fig. 2, is connected to the $\varphi$-OTDR based DAS system for interrogation. 


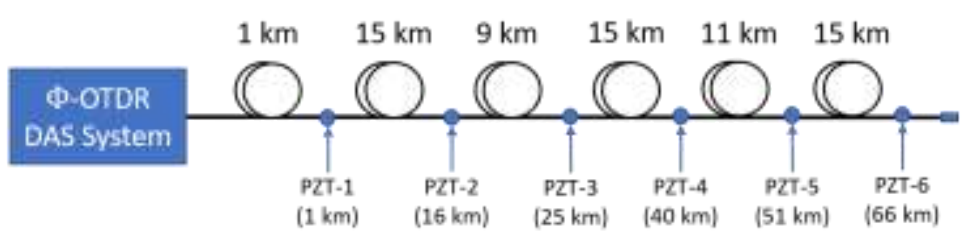

Fig. 2. PZT test set-up.

The tests are performed by analyzing the PZT vibrations of $2 \mathrm{Vpp}$ at $200 \mathrm{~Hz}$. The datasets are collected for two cases of DAS scheme, i.e. the default case (without EVOA) and the proposed EVOA case. The $\varphi$-OTDR systems suffer from fading phenomena on the return signal levels, which results in time varying SNR values. In this work, for this reason, we have used mean SNR values for comparison instead of single SNR values, which is explained in detail and applied in [6] and [7]. The mean SNRs are calculated at six PZT positions and they are compared in Fig. 3. In this figure, blue and yellow lines represent the data collected from the default scheme (without EVOA) and the proposed EVOA scheme with a square signal having a positive pulse width of $150 \mu$ s (Fig. 1b inset).

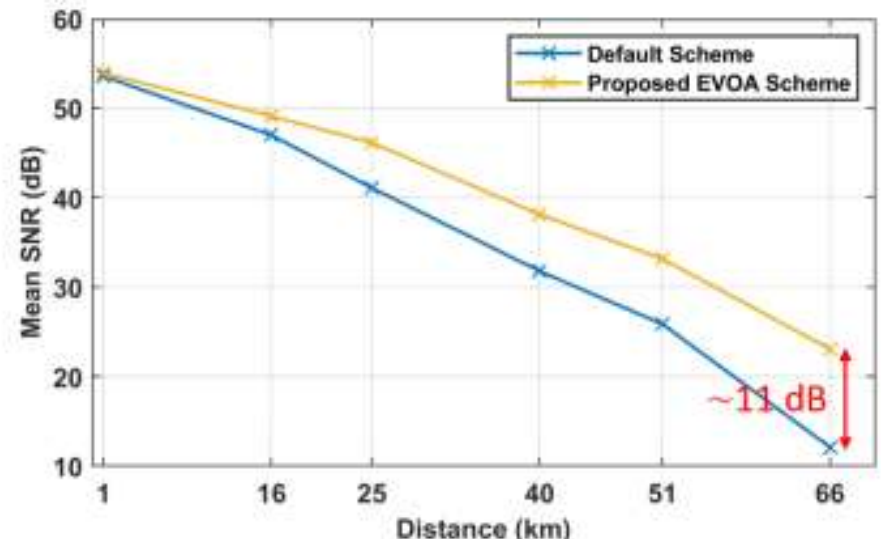

Fig. 3. Mean SNRs calculated at six PZT positions for two cases of DAS scheme.

The results show that at the $2^{\text {nd }}, 3^{\text {rd }}, 4^{\text {th }}, 5^{\text {th }}, 6^{\text {th }}$ PZT positions, i.e. at the distances of $16 \mathrm{~km}, 25 \mathrm{~km}, 40 \mathrm{~km}, 51$ $\mathrm{km}$, and $66 \mathrm{~km}$ the proposed scheme provides higher mean SNR than the default scheme. The mean SNR difference increases as the distance goes up and reaches approximately $11 \mathrm{~dB}$ at the distance of $66 \mathrm{~km}$. Complying with expectations, at the $1^{\text {st }}$ PZT position, i.e. at the distance of $1 \mathrm{~km}$, the default scheme provides similar SNR with the proposed scheme due to added attenuation at close-in distances in order to prevent saturation. Starting from the $2^{\text {nd }}$ PZT position, i.e. at the distances above $16 \mathrm{~km}$, SNRs obtained from the proposed scheme prevail. The results also imply that the proposed scheme would be able to detect the signals with a significant $\mathrm{dB}$ above the noise floor at farther away distances above $66 \mathrm{~km}$ where the default scheme would lie under noise floor.

\section{Conclusion}

A practical and effective method for extending the range and SNR performance of the $\varphi$-OTDR DAS system is proposed by utilizing an electronic variable attenuator. SNR improvement of the proposed method is experimentally verified with an ever-increasing mean SNR difference, reaching $\sim 11 \mathrm{~dB}$ at the distance of $66 \mathrm{~km}$. The method has the potential for several applications which require a simple and cost-effective solution for long range applications.

\section{References}

[1] X. Liu, B. Jin, Q. Bai, Y. Wang, D. Wang, Y. Wang, “Distributed Fiber-Optic Sensors for Vibration Detection,” Sensors 16(12), 1164 (2016).

[2] L. Lv, F. Pang, H. Liu, X. Mei, J. Chen, Y. Li, Y. Song, and T. Wang, "50-km-Long distributed vibration fiber sensor based on phasesensitive OTDR using coherent detection," in CLEO-PR, (Institute of Electrical and Electronics Engineers, New York, 1973).

[3] Jin Li, Zinan Wang, Li Zhang, Fei Peng, Shunkun Xiao, Huijuan Wu, and Yunjiang Rao "124km phase-sensitive OTDR with Brillouin amplification", in Proc. SPIE 9157, 23rd International Conference on Optical Fibre Sensors, 91575Z (SPIE, Washington, D.C.)

[4] F. Peng, H. Wu, X.-H. Jia, Y.-J. Rao, Z.-N. Wang, and Z.-P. Peng, "Ultra-long high-sensitivity $\Phi-O T D R$ for high spatial resolution intrusion detection of pipelines," Optics Express, Vol. 22, No. 11 (2014).

[5] Z. N. Wang, et al., "Ultra-long phase-sensitive OTDR with hybrid distributed amplification," Optics Letters, Vol. 39, No. 20 (2014).

[6] F. Uyar, T. Kartaloglu, I. Ozdur, E. Ozbay, "Field test and fading measurement of a distributed acoustic sensor system over a 50 km-long fiber," in Proc. SPIE 10654, Fiber Optic Sensors and Applications XV, 106540D (SPIE, Washington, D.C.).

[7] F. Uyar, T. Onat, C. Unal, T. Kartaloglu, E. Ozbay, and I. Ozdur, "A Direct Detection Fiber Optic Distributed Acoustic Sensor With a Mean SNR of $7.3 \mathrm{~dB}$ at 102.7 km,” IEEE Photonics Journal, vol. 11, no. 6, pp. 1-8, 2019. 